\title{
Heart Valve Lesions Due to the Formation of a Beta-Hemolytic Streptococ Role of Adhesion Molecules
}

\author{
Semsi Altaner ${ }^{1}$, Turhan Kurum ${ }^{2}$, Muzaffer Demir $^{3}$, Burhan Turgut ${ }^{4}$, Turan Ege ${ }^{5}$, Enver Duran ${ }^{5}$ \\ ${ }^{1}$ Pathology Department, School of Medicine, Baskent University, Istanbul, Turkey \\ ${ }^{2}$ TEM Hospital, Istanbul, Turkey \\ ${ }^{3}$ Hematology Department, School of Medicine, Trakya University, Edirne, Turkey \\ ${ }^{4}$ School of Medicine, Namik Kemal University, Tekirdag, Turkey \\ ${ }^{5}$ School of Medicine, Trakya University, Edirne, Turkey \\ Email: semsialtaner@hotmail.com
}

Received June 8, 2013; revised July 9, 2013; accepted July 18, 2013

Copyright (C) 2013 Semsi Altaner et al. This is an open access article distributed under the Creative Commons Attribution License, which permits unrestricted use, distribution, and reproduction in any medium, provided the original work is properly cited.

\begin{abstract}
Objectives: A rise in the levels of adhesion molecules such as VCAM-1, ICAM-1and E-selectin in valve disease patients has been reported lately. In our study, by detecting the presence of adhesion molecule expression in the valve endothelium we will try to show the level of adhesion molecules in peripheral blood leucocytes. Materials and Methods: Valve samples were obtained from patients having undergone aortic and mitral valve replacement due to symptomatic aortic stenosis/aortic insufficiency and/or mitral stenosis/mitral insufficiency. The clinical preoperative diagnosis was made using two-dimensional echocardiography and Doppler echocardiography. Rheumatic valves were in group B $(\mathrm{n}=$ 20). Group A $(\mathrm{n}=8)$ constituted the control group. Immunohistochemical staining was performed using CD4, CD8, CD54/ICAM-1, and CD106/VCAM-1. Flow cytometric analysis was performed. The Kolmogorov-Smirnov test and Fisher's exact test were used for the comparison of categorical variables. Results: Group A (non-rheumatic) patients were found to be older than group B (rheumatic) patients $(59.8 \pm 11.4$ years vs. $45.3 \pm 11.8$ years, $p=0.008)$. In group B VCAM-1 level was higher than that of group A $(296.6 \pm 21.2$ vs. $258.5 \pm 42.1, \mathrm{p}=0.004)$. CD1 lb monocyte in group $\mathrm{B}$ was higher than in group A $(98.8 \pm 0.5$ vs. $92.9 \pm 9.7, \mathrm{p}=0.003)$. CD11b granulocyte was higher in group B than in group A $(99.96 \pm 0.05$ vs. $93.79 \pm 13.26, p=0.33)$. Significant differences were not determined in the other parameters. Conclusion: The fact that increases in serum VCAM-1 and CD-11b only occurred in patients with rheumatic valvular disease in our study suggests that inflammation in patients with the same hemodynamic disorder is higher in rheumatic valvular disease than in the ones with non-rheumatic valvular disease.
\end{abstract}

Keywords: Rheumatic Fever; Chronic Valve Disease; Cell Adhesion Molecules

\section{Introduction}

Rheumatic fever (RF) is an inflammatory disease where the immune mechanism targets the heart following Group A beta-hemolytic streptococcal pharyngitis $[1,2]$. Immune response developing against streptococcal antigens in the development of RF has been accepted as a cause of initiation of the disease. Both genetic and environmental factors are effective in the development of RF. RF mostly reveals itself by leading to carditis. Pathological signs of rheumatic carditis, which include Anitschkow myocytes and Aschoff nodules, have an effect on heart tissue $[3,4]$. Valve involvement is diagnosed by observing the development of verru and nodules on the mitral valve, accompanied by edema and cellular inflammation.
The approach accepted during the past half-century has appeared to be that Group A streptococcal antigens may break immune tolerance in vivo as a consequence of antigenic similarity and/or an abnormal response to this bacterial antigen [5]. Although factors that precede the progression of fibrosis in some patients with rheumatic fever have not been entirely revealed, progressive fibrosis of valves and chronic valve disease develop in such patients. The pathogenetic mechanisms causing RF and non-rheumatic heart disease could still not be entirely determined [5].

Rheumatic heart disease is the most frequent cause of valvular diseases in developing countries [5]. Rheumatic fever and non-rheumatic heart diseases are still an important worldwide public health problem in terms of car- 
diovascular mortality and morbidity in the 21 st century [6]. Chronic rheumatic valvular disease is considered to be a late sequela that occurs in $30 \%$ of patients with rheumatic fever. Mitral valvulitis is the most common lesion of acute rheumatic carditis. Valvulitis generally occurs in the valves and chordae tendineae due to fibrous tissue accumulation as a consequence of recovery and disease episodes. Rheumatic fever is diagnosed in $99 \%$ of patients who undergo mitral valve replacement due to mitral valve stenosis [6].

There is a period of at least 2 years and generally long intervals (10 to 20 years) between the occurrence of rheumatic carditis attack and symptomatic valve disease $[7,8]$. Primary lesions of the valve in RF patients are translucent nodules observed along the closure lines of the valve. The valve weakens and has a fibrotic and calcified appearance due to the development of valvulitis. Mitral valvulitis occurring due to an attack of RF causes abnormal flow across the valve. This changing flow pattern develops an increasing tension on the disordered structure of the valve. In addition, inflammation in the valve leads to the development of fibrin deposits on the surface of the valve which causes an increase in the abnormal flow pattern across the valve. Tension and pressure exerted over the course of years results in weakness and fibrosis of the valve. Anatomical differences in patients of severe mitral stenosis (MS) or aortic stenosis (AS) are developed by the stable and progressive trauma caused by the turbulent flow [9]. It is still discussed whether the development of anatomical differences in the valve are of a progressing rheumatic origin or progresssive fibrosis, weakening and calcification developing in the valve which is deformed by a turbulent flow. The endothelial cells in the walls of large vessels, such as the aorta, and cardiac valves are exposed to high shear stress. Cardiac valve endothelial cells have a special morphological structure. These valves are in the form of surface microvilli [10].

Cell adhesion molecules are special structures found on the surface of circulating leucocytes and endothelial cells, which regulate the migration of leucocytes to the functionally different body tissues. These cell adhesion molecules provide the orientation of effectors cells to the sites of inflammation and tissue destruction, lymphocyte circulation among lymphoid tissues and the progenitor cell development and maturity within the hematopoietic microenvironment [11,12]. Cell adhesion molecules in human serum in healthy patients can be detected at very low levels. However, there is also a group of diseases with inflammatory or vascular etiology for which low levels of cell adhesion molecules can be detected.

Intercellular adhesion molecule-1 (ICAM-1), vascular adhesion molecule-1 (VCAM-1) and E-selectin are structures functioning as receptors for the inflammatory cells which are detected circulating on the vascular endothelium. ICAM is a membrane glycoprotein which allows glycolysis at different values [13,14]. Endothelial cells are situated on the fibroblasts, epithelial cells and active lymphocytes. VCAM-1 are structures activated by Interleukin-1B (IL), TNF-alpha and IL-4 situated primarily on bone marrow fibroblasts, macrophages, synovial lining cells, germinal center dendritic cells and endothelial cells [15]. Selectins are structures which have a very important role in the first contact and rolling of leucocytes on the active endothelium. E-selectin is a molecule found on endothelial cells stimulated only by cytokines $[13,14]$. The increased serum levels of this molecule are accepted as an indicator of endothelial cell activation. Unlike other adhesion molecules which have a wider tissue distribution, E-selectin is found only on the activated endothelium. A rise in E-selectin level will show endothelial activation as a component of a special pathology or damage. In vivo expression generally appears along with active granulomatous inflammation and delayed hypersensitivity.

CD11b (Mac-1 $\alpha$ chain) is one of the $\beta 2$-integrins expressed on leucocytes. It mediates leukocyte adhesion and transmigration to the endothelial cells. In addition, it has a role in neutrophil adhesion (homotopical adhesion) and chemotaxis.

A rise in adhesion molecule levels, such as VCAM-1, ICAM-1 and E-selectin in valve disease patients has been reported recently [16-18]. In our study by detecting the presence of CD11b lymphocytes in the valve tissue, adhesion molecule expression in the valve endothelium and the level of adhesion molecules in peripheral blood leucocytes, we will try to show whether there is an active inflammatory process during the chronic period of rheumatic fever. On the condition of the presence of the process we will try to differentiate whether a progressive inflammation or hemodynamic loading caused by underlying preload and after load increase leads to an increase in adhesion molecules by applying the findings at tissue level.

\section{Materials and Methods}

\subsection{Tissues}

Valve samples were obtained from patients having undergone aortic and mitral valve replacement due to symptomatic aortic stenosis/aortic insufficiency and/or mitral stenosis/mitral insufficiency in Trakya University Hospital for histopathological examination. The clinical preoperative diagnosis was made using two-dimensional echocardiography and Doppler echocardiography. Calcified and/or fibrotic rheumatic valves were collected during the operations. (Group B; $\mathrm{n}=20,11$ male, 9 female). Mitral valve tissue evaluated as ischemic during valve 
repair surgery and aortic valve tissue of non-rheumatic etiology (Group A; $\mathrm{n}=8$, 5 male, 3 female) constituted the control group. Patients with malignity, inflammatory disease, renal or liver disease, diabetes mellitus, hypertension, hyperlipidemia, deep venous thrombosis, pulmonary emboli or a past history of surgery in both groups were excluded from the study. Whether or not calcification was present, commissural fusion and fibrous thickening within the site of valve closures and revascularization were evaluated as the macroscopic evidence of rheumatic origin.

\subsection{Immunohistochemical Study}

All tissue samples were fixed with $10 \%$ neutral formalin and embedded in paraffin. 3- $\mu \mathrm{m}$ thick sections were obtained from paraffin blocks. First, the sections were dehydrated in series of alcohol then they were placed in a $10 \mathrm{mM}$ citrate tampon and were exposed to antigen retrieval for 40 minutes. After heating, the sections were left to cool at room temperature and they were washed with tampon saline. They were treated with $3 \%$ hydrogen peroxide in methanol for 5 minutes. Immunohistochemical marking was performed using a streptavidin-biotin peroxidase system (DAKO LSAB kit, United Kingdom). CD4 Ab-8 (clone 4B12, Neomarkers, United Kingdom), CD8 Ab-7 (clone 4B11, Neomarkers, United Kingdom), CD54/ICAM-1 Ab-4 (Clone 54CO4, Neomarkers, United Kingdom), and CD106/VCAM-1 Ab-3 (Clone 1.4 C3, NeoMarkers, United Kingdom) were incubated for an hour at room temperature. Secondary antibodies were performed for 15 minutes after rinsing with a saline phosphate tampon. The sections were incubated with streptavidin-biotin complex for 10 minutes and treated for 5 minutes with a hydrogen peroxide phosphate tampon. They were colored with 3.3 diaminobenzidine tetrahydrochloride (DAB). Mayer's hematoxylin was performed as counterstaining. Afterwards, the sections were dehydrated with a series of ethanol performance. Cytoplasmatic and membranous staining was accepted as positive for ICAM-1 and VCAM-1.

\subsection{Cytokins}

Blood samples were obtained in the fasting state between 9 and 10 am in the control and study groups in order to be able to exclude the possible influence of circadian fluctuation. Blood samples were collected in 5-ml tubes containing 3.8\% Na-citrate. Blood samples were obtained under minimal tourniquet pressure from the antecubital vein using a sterile 22 -gauge needle syringe in a single attempt, from the patients in the supine position for at least 20 minutes and the samples were centrifuged at 3000 turnover for 10 minutes at $+4^{\circ} \mathrm{C}$. Plasma was decomposed and frozen at $-80^{\circ} \mathrm{C}$. Blood samples were analyzed according to standard laboratory methods including whole blood count, biochemical and electrolyte measurements, and blood samples with excessive hemolysis were excluded from the study. The soluble VCAM1 and ICAM-1 concentrations of human plasma samples were measured by using commercially available, solidphase, enzyme-linked kits (Diaclone; Besancon, France). The blood samples were processed according to the instructions of the manufacturer. The duration between the collection of blood samples and antibody labeling was standardized as an hour. Anti-VCAM-1 and anti-ICAM-1 antibodies were sequentially added after the testing samples were treated with monoclonal antibodies found in the standard of known soluble concentrations of VCAM1 and ICAM-1. Unbound materials were removed and an enzyme (streptavidin-peroxidase) was added after an hour of incubation at room temperature. Subsequent to incubation and washing to remove the entire unbound enzyme, a substrate solution (chromogen [tetramethylbenzidine]) was added to induce a color reaction. The color reaction was measured using a microplate reader (Dynex Technologies; Chantilly, UK) and reading the absorbance at $450 \mathrm{~nm}$ with a correction wavelength of $630 \mathrm{~nm}$. A standard curve was obtained using the mean absorbance values of VCAM-1 and ICAM-1, and the VCAM-1 and ICAM-1 concentrations in unknown plasma samples were calculated by linear regression. All standards and samples were tested in duplicate. According to the manufacturer of the kits, the minimum quantifiable doses of VCAM-1 and ICAM- 1 are $<0.6 \mathrm{ng} / \mathrm{ml}$ and $<0.1 \mathrm{ng} / \mathrm{ml}$ respectively.

\subsection{Cell Preparation, Marking and Flow Cytometric Analysis}

Fluorescein isothiocyanate (FITC), phycoerythrin (PE) and phycoerythrin-cyanin 5.1 (PC5) dyes with conjuge monoclonal antibodies isotophic controls were used in the study carried out with three colors through direct immunofluorescence method.

Preparing the samples: The blood sample with $100 \mu \mathrm{l}$ EDTA was treated in a suitable way and incubated with a monoclonal antibody cocktail at room temperature and in darkness for 20 minutes. In addition to this, lysis of erythrocytes and fixation of leukocytes were carried out in a multi-Q prep workstation, Beckman Coulter, which was an automated device for preparing samples. Analysis was conducted using a coulter Epics XL current cytometry device. Expression rates of antigens on lymphocytes, determined according to light characteristics formed on the histogram with system II software, were measured.

\subsection{Statistics}

Continuous variables were described in terms of the 
mean $\pm \mathrm{SD}$ and categorical variables were described as percentages. The suitability of the continuous variables with normal distribution was examined using the Kolmogorov-Smirnov test. In comparing variables between the groups, a T-test was used for independent groups with normal distribution but the Mann-Whitney U test was used for independent groups without normal distribution. Fisher's exact test was used for the comparison of categorical variables. $\mathrm{P}<0.05$ was considered statisticcally significant.

\section{Results}

The clinical characteristics for each patient are summarized in Table 1. Group A patients were found to be older than group B patients $(59.8 \pm 11.4$ years vs. $45.3 \pm$ 11.8 years, $p=0.008$ ). In group B the ICAM-1 level was higher than that of group A $(296.6 \pm 21.2$ vs. $258.5 \pm$ $42.1, \mathrm{p}=0.004)$. Group B had higher monocyte CD11b levels in group A $(98.8 \pm 0.5$ vs. $92.9 \pm 9.7, \mathrm{p}=0.003)$. CD11b granulocyte level was higher in group B group than in group A $(99.96 \pm 0.05$ vs. $93.79 \pm 13.26, p=$ 0.33 ). Significant differences were not determined in other parameters. No significant correlation between the parameters of RVH and non-RVH could be found with immunohistochemistry. No difference was found between gender and the diagnosis of RVH and non-RVH.

The light microscopic examination with hematoxylin eosin revealed that the valves were observed to consist of fibro-hyalinized tissues in wide sites. Some of the heart valves exhibited histiocyte and lymphocyte infiltration and in some of them young connective tissue proliferation existed. In all heart valves there were small-sized vascular structures which were not so intense. There were no differences in both groups at the light microscopic level.

In immunohistochemical studies, CD4 or CD8 cytoplasmic positivity was detected in some of the lymphocytes, making clusters in patches located in valvular stroma. In some lymphocytes, fibroblasts and endothelial cells, rare CD54 (ICAM-1) cytoplasmic positivity was detected. CD106 (VCAM-1) positivity was observed in vascular endothelial cells and some fibroblasts.

\section{Discussion}

Rheumatic fever is a multi-systemic inflammatory disease occurring following group A beta-hemolytic strep

Table 1. CD4, CD8, ICAM-1 and VCAM-1 distribution of non-rheumatic and rheumatic groups.

\begin{tabular}{ccccc}
\hline & CD4 & CD8 & ICAM-1 & VCAM-1 \\
\hline (Group A (non-rheumatic) $(\mathrm{n}=8)$ & 1 & 1 & 1 & 2 \\
Group B (rheumatic) $(\mathrm{n}=20)$ & 7 & 5 & 5 & 10 \\
\hline
\end{tabular}

toccal pharangitis and it commonly affects the heart. It is also triggered by immunological mechanisms. Recurrent streptococcal pharyngeal infections after a previous attack increases the reactivation risk of the disease. The number of lymphocytes causes an increase both in cardiac valves and in peripheral blood and also the cytokine levels in blood increase during acute rheumatic carditis.

In this course, adhesion molecules play a considerable role. Intracellular adhesion molecules (ICAM-1), vascular cell adhesion molecules (VCAM-1) and E-selectin exist in the vascular endothelium and act as ligands for surface receptors of circulating inflammatory cells [11, 13-15].

It is known that adhesion molecules differentiate their antigenic expression according to their activation status and location $[19,20]$. Detailed pathological investigations researching the circulating forms of VCAM-1, ICAM-1 and E-selectin in the plasma have revealed the increased expression of cellular adhesion molecules in endothelial cells and other tissue types [21-23].

Yetkin et al. compared 34 patients who underwent balloon mitral valvuloplasty with 34 healthy people participating as the control group [18]. In their study the serum levels of E-selectin, ICAM-1 and VCAM-1 were found to be elevated in patients with mitral stenosis compared with the control group. According to this result, they proposed that E-selectin, ICAM-1 and VCAM-1, which were the elevated serum adhesion molecules, are indicators of ongoing chronic inflammation in patients with significant rheumatic mitral stenosis. The researchers subsequently examined the values in the patient group before and after the procedure and found that after PMBV procedure, VCAM values were elevated, E-selectin values had decreased and ICAM remained unchanged. They proposed with respect to this result that ICAM-1, VCAM-1 and E-selectin exhibit discrete changes as a response after percutaneous mitral balloon valvuloplasty [24]. They indicated that further investigations should be required to clarify the mechanism of the relationship between adhesion molecules and PMBV in addition to rheumatic mitral stenosis. Chen et al. proposed that serum VCAM-1 level decreased significantly after PMBV and elevated plasma VCAM-1 level was related to hemodynamic abnormality, not rheumatic activity [25].

Shahi et al. determined that soluble adhesion molecules such as ICAM-1, VCAM-1 and E-selectin are elevated in non-rheumatic aortic valvular stenoses. The authors proposed that the pathogenesis of non-rheumatic aortic stenosis which had been believed to be degenerative actually had an inflammatory and immunologic etiology. No study investigating the tissue and serum levels of cell adhesion molecules of patients with rheumatic aortic valvular disease was detected in literature. 
In the above mentioned studies because no immunochemical studies were performed on the valvular tissues of patients with mitral or aortic valvular diseases, and because the relationship between serum levels was not investigated, it could not established whether these findings resulted from hemodynamic disorders or from degenaration. We determined that VCAM-1 in serum levels increased in patients with rheumatic valvular disease compared to the ones with non-rheumatic valvular disease. No significant difference was found in other adhesion molecules from the serum examination. Hence, we assume that hemodynamic disorders may have an increasing effect on cell adhesion molecules in addition to inflammation.

Roberts et al. tried to clarify the immunity-mediated mechanism in rheumatic heart disease caused by group A streptococcal infection by analyzing anti-CD4, anti-CD8 and VCAM-1 in valvular tissues acquired from 6 patients with rheumatic valvular disease requiring valvular replacement (2). Out of the 6 patients, only 2 had an endothelium suitable for examination. They detected that CD4 and CD8 lymphocytes penetrated through the subendothelial layer on the valve surface and attached to the valve endothelium. They proposed that $\mathrm{T}$ cell extravasation through the surface endothelium into the valve seemed to be an important process in rheumatic heart disease development. The researchers anticipate that lymphocytic infiltration through valve surface endothelium is a potential triggering step in disease pathogenesis. However, there was no information about the serum values of the patients in this study. Mitral valves taken from 3 in order to control autopsy subjects were studied. As for our study in the same patient group with mitral stenosis and/or mitral failure and/or aort stenosis and/or aort failure, examination was performed for adhesion molecules both in valvular tissue and soluble adhesion molecules in blood. However, in our study, ICAM-1, VCAM1 and E-selectin were analyzed using IHK method both in patients with ischemic and degenerative valvular disease and in the ones with rheumatic valvular disease, and no significant difference was found.

Ghaisas et al. studied ICAM-1, VCAM-1 and E-selectin expression via immunohistochemical method in patients with non-rheumatic aortic disease requiring aortic valvular replacement. They also analyzed the serum values before and after surgery. They reported that adhesion molecules located in the tissues increased. E-selectin increased both in serum and tissue. As a conesquence, they proposed that the expression of adhesion molecules in the involved valves supported the inflamematory component theory in degenerative aortic valvular disease and this was the main source for E-selectin located in the serum [17].

Muller et al. investigated the expression of cell adhe- sion molecules using immunohistochemical method in degenerative cardiac valves and the endothelial cells of heart valves with endocarditis [26]. As expected, ICAM$1, \mathrm{CD} 34$ and CD31 were found both in degenerated and inflamed valves. Muller et al. detected that significant expressions of E-selectin and VCAM-1 were not only in inflamed cardiac valves but also in the majority of degenerated valves not exhibiting the morphological signs of inflammation. They thought that they could present this as an attractive finding to explain why fibrotic cardiac valves were prone to recurrent endocarditis. Also, they proposed that further investigation was required to discover why E-selectin and VCAM-1 which are endothelial activation markers were also expressed in degenerated cardiac valves [26].

Wallby et al. investigated T lymphocyte infiltration by comparing the patients with degenerative calcification of tricuspid aortic valves to patients with congenital bicuspid aortic valve secondary calcification [27]. Consequently without considering valvular anomaly, by determining $\mathrm{T}$ lymphocyte infiltration in acquired aortic stenosis equally, they proposed that $\mathrm{T}$ lymphocyte infiltration should be regarded in inflammation pathogenesis.

Finally, none of these studies examined or compared rheumatic and non-rheumatic valvular disease in terms of serum and tissue values. That only serum VCAM-1 and tissue CD11b increases occurred in our study in patients with rheumatic valvular disease suggests that inflammation in patients with the same hemodynamic disorder is higher in rheumatic valvular disease than in the ones with non-rheumatic valvular disease.

Restrictions: The values for rheumatic and ischemic valvular diseases were not compared with the normal control group. Average values were above the cut-off level. In addition, control values were not examined after valvular changes. For this reason, whether hemodynamic improvement occurred or not cannot be shown with the serum values.

\section{REFERENCES}

[1] M. W. Cunningham, "Pathogenesis of Group A Streptoccal Infections," Clinical Microbiology Reviews, Vol. 13, No. 3, 2000, pp. 470-511. doi:10.1128/CMR.13.3.470-511.2000

[2] S. Roberts, S. Kosanke, S. T. Dunn, D. Jankelow, M. G. C. Duran and M. W. Cunningam, "Pathogenetic Mechanism in Rheumatic Carditis: Focus on Valvular Endothelium," The Journal of Infectious Diseases, Vol. 183, No. 3, 2001, pp. 507-511. doi:10.1086/318076

[3] G. Murphy, "The Characteristic Rheumatic Lesions of Striated and of Non-Striated or Smooth Muscle Cells of the Heart," Medicine (Baltimore), Vol. 42, No. 2, 1963, pp. 73-118. doi:10.1097/00005792-196303000-00001

[4] G. H. Stollermann, "Rheumatic Fever and Streptococcal 
Infection," Grune and Stratton, New York, 1975.

[5] A. Saxena, "Rheumatic Fever and Long-Term Sequelae in Children," Current Treatment Options in Cardiovascular Medicine, Vol. 4, No. 4, 2002, pp. 309-319. doi:10.1007/s11936-002-0011-7

[6] World Health Organization, "Rheumatic Fever and Rheumatic Heart Disease," Report of an Expert Panel, Geneva, 2004.

[7] J. C. Rowe, F. Bland, H. B. Sprague and P. D. White, "Course of Mitral Stenosis without Surgery: 10 and 20 Years Perspectives," Annals of Internal Medicine, Vol. 52, No. 4, 1960, pp. 741-749. doi:10.7326/0003-4819-52-4-741

[8] E. L. Kaplan, "Pathogenesis of Acute Rheumatic Fever and Rheumatic Heart Disease: Evasive after Half a Centruy of Clinical, Epidemiological, and Laboratory Investigation," Heart, Vol. 91, 2005, pp. 3-4. doi:10.1136/hrt.2004.034744

[9] L. L. Minich, L. Y. Tani, L. T. Pagotto, R. E. Shaddy and L. G. Veasy, "Doppler Echocardiography Distinguishes between Silent Mitral Regurgitation in Patients with Rheumatic Fever," Clinical Cardiology, Vol. 20, No. 11, 1997, pp. 924-926. doi:10.1002/clc.4960201105

[10] W. Doerr, W. Giese, L. D. Leder and W. Remmele, "Organpathologie, Band I: Herz und Gefäße, Blut und Blutbereitende Organe, Atemwege und Lungen," Georg Thieme Verlag, Stuttgart, 1974.

[11] S. J. Weiss, "Tissue Destruction by Neutrophils," The New England Journal of Medicine, Vol. 320, No. 6, 1989, pp. 365-376. doi:10.1056/NEJM198902093200606

[12] H. A. Papadaki, "Cell Adhesion Molecules in Haematology," Haema, Vol. 2, 1999, pp. 180-191.

[13] T. A. Springer, "Adhesion Receotors of the Immune System," Nature, Vol. 346, No. 6283, 1990, pp. 425-434. doi: $10.1038 / 346425 \mathrm{a} 0$

[14] R. Piggot and W. Newman, "Circulating Adhesion Molecules in Disease," Immunology Today, Vol. 14, No. 10, 1993, pp. 506-512. doi:10.1016/0167-5699(93)90267-O

[15] M. J. Elices, L. Osborn, Y. Takada, C. Crouse, S. Luhowskj, M. E. Hemler and R. R. Lobb, "VCAM-1 on Activated Endothelium Interacts with the Leucocytes Integrin VLA-4 at the Site Distinct from VLA-4/Fibronectin Binding Site," Cell, Vol. 60, No. 4, 1990, pp. 577-584. doi:10.1016/0092-8674(90)90661-W

[16] C. H. Shahi, N. K. Ghasias, M. Goggins, B. Foley, P. Crean, D. Kelleher and M. Walsh, "Elevated Levels of Circulating Soluble Adhesion Molecules in Patients with Nonrheumatic Aortic Stenosis," American Journal of Cardiology, Vol. 79, No. 7, 1997, pp. 980-982. doi:10.1016/S0002-9149(97)00027-1

[17] N. K. Ghaisas, J. B. Foley, D. S. O'Briain, P. Crean, D. Kelleher and M. Walsh, "Adhesion Molecules in Nonrheumatic Aortic Valve Disease: Endothelial Expressio, Serum Levels and Effects of Valve Replacement," Journal of the American College of Cardiology, Vol. 36, No. 7, 2000, pp. 2257-2262. doi:10.1016/S0735-1097(00)00998-0
[18] E. Yetkin, A. R. Erbay, M. Ileri, H. Turhan, M. Balci, S. Çehreli, G. Yetkin and D. Demirkan, "Levels of Circulating Adhesion Molecules in Rheumatic Mitral Stenosis," American Heart Journal, Vol. 88, No. 10, 2001, pp. 1209-1211.

[19] N. Patey, P. Lesavre, L. Halbwaschs-Macarelli and L. H. Noel, "Adhesion Molecules in Human Cresentric Glomerulojnephritis," The Journal of Pathology, Vol. 179, No. 4, 1996, pp. 414-420.

doi:10.1002/(SICI)1096-9896(199608)179:4<414::AID-P ATH601>3.0.CO;2-J

[20] B. I. Tropea, P. Huie, J. P. Cooke, P. S. Tsao, R. K. Sibley and C. K. Zarins, "Hypertension-Enhanced Monocyte Adhesion in Experimental Atherosclerosis," Journal of Vascular Surgery, Vol. 23, No. 4, 1996, pp. 596-605. doi:10.1016/S0741-5214(96)80038-3

[21] A. H. J. Gearing and W. Newman, "Circulating Adhesion Molecules in Disease," Immunology Today, Vol. 14, 1993, pp. 506-512. doi:10.1016/0167-5699(93)90267-O

[22] C. M. Ballantyne, E. A. Mainolfi, J. B. Young, N. T. Windsor, B. Cocanougher, E. C. Lawrence, M. S. Polack, M. L. Entman and R. Rothlein, "Relationship of Increased Levels of Circulating Intercellular Adhesion 1 after Heart Transplantation to Rejection: Human Leucocyte Antigen Mismatch and Survival," The Journal of Heart and Lung Transplantation, Vol. 13, No. 4, 1994, pp. 597-603.

[23] K. Nakai, C. Itoh, K. Kawazoe, Y. Miura, H. Sotyanagi, K. Hotta, T. Itoh, J. Kamata and K. Hiramori, "Concentration of Soluble Vascular Adesion Molecule-1 Correlated with Expression of VCAM-1 mRNA in Human Atherosclerotic Aorta," Coronary Artery Disease, Vol. 6, No. 6, 1996, pp. 497-502.

[24] E. Yetkin, A. R. Erbay, H. Turhan, M. Balci, F. Yetkin, G. Yetkin, M. Ileri, K. Senen, R. Atak and S. Cehreli, "Changes in Plasma Levels of Adhesion Molecules after Percutaneous Mitral Balloon Valvuloplasty," Cardiovascular Pathology, Vol. 13, No. 2, 2004, pp. 103-108. doi:10.1016/S1054-8807(03)00128-5

[25] M. C. Chen, H. W. Chang, S. S. Juang, H. K. Yip, C. J. $\mathrm{Wu}, \mathrm{T} . \mathrm{H} . \mathrm{Yu}$ and C. I. Cheng, "Percutaneous Transluminal mitral Valvuloplasty Reduces Circulating Vascular Cell Adhesion Molecule-1 in Rheumatic Mitral Stenosis," Chest, Vol. 125, No. 4, 2004, pp. 1213-1217. doi:10.1378/chest.125.4.1213

[26] A. M. Muller, C. Cronen, L. I. Kupferwasser, H. Oelert, K. M. Muller and C. J. Kirkpatrick, "Expression of Endothelial Cell Adhesion Molecules on Heart Valves: Up-Regulation in Degeneration As Well As Acute Endocarditis," The Journal of Pathology, Vol. 191, No. 1, 2000, pp. 54-60. doi:10.1002/(SICI)1096-9896(200005)191:1<54::AID-P ATH568>3.0.CO;2-Y

[27] L. Wallby, B. Janerot-Sjöberg, T. Steffensen and M. Broqvist, "T Lymphocyte Infiltration in Non-Rheumatic Aortic Stenosis: A Comparative Descriptive Study between Tricuspid and Bicuspid Aortic Valves," Heart, Vol. 88, 2002, pp. 348-351. doi:10.1136/heart.88.4.348 